\title{
THE OXFORD HANDBOOK OF
}

\section{LINGUISTIC ANALYSIS}

\author{
Edited by \\ BERND HEINE \\ and \\ HEIKO NARROG
}




\section{OXFORD \\ UNIVERSITY PRESS}

Great Clarendon Street, Oxford ox2 6DP

Oxford University Press is a department of the University of Oxford.

It furthers the University's objective of excellence in research, scholarship, and education by publishing worldwide in

Oxford New York

Auckland Cape Town Dar es Salaam Hong Kong Karachi

Kuala Lumpur Madrid Melbourne Mexico City Nairobi New Delhi Shanghai Taipei Toronto

With offices in

Argentina Austria Brazil Chile Czech Republic France Greece

Guatemala Hungary Italy Japan Poland Portugal Singapore South Korea Switzerland Thailand Turkey Ukraine Vietnam

Oxford is a registered trade mark of Oxford University Press in the UK and in certain other countries

Published in the United States

by Oxford University Press Inc., New York

(c) 2010 editorial matter and organization Bernd Heine and Heiko Narrog (C) 2010 the chapters their various authors

The moral rights of the authors have been asserted

Database right Oxford University Press (maker)

First published 2010

All rights reserved. No part of this publication may be reproduced, stored in a retrieval system, or transmitted, in any form or by any means, without the prior permission in writing of Oxford University Press,

or as expressly permitted by law, or under terms agreed with the appropriate reprographics rights organization. Enquiries concerning reproduction outside the scope of the above should be sent to the Rights Department,

Oxford University Press, at the address above

You must not circulate this book in any other binding or cover and you must impose the same condition on any acquirer

British Library Cataloguing in Publication Data

Data available

Library of Congress Cataloging in Publication Data

Data available

Typeset by SPI Publisher Services, Pondicherry, India Printed in Great Britain on acid-free paper by

CPI Antony Rowe, Chippenham, Wiltshire

ISBN 978-0-19-954400-4

13579108642 


\title{
DEPENDENCY \\ GRAMMAR AND VALENCY THEORY
}

\author{
VILMOS Á GEL \\ KLAUS FISCHER
}

\subsection{CENTRAL QUESTIONS}

\subsubsection{Contextualization}

THE best way to introduce a reader to dependency grammar (DG) and valency theory (VT) - both come out of the same research tradition-is to present the syntactic theory of Lucien Tesnière, which is still relevant today (section 10.1). In the following sections we will give an overview of the development, current state, and possible future concerns first of VT (section 10.2), then of DG (section 10.3). However, this chapter can only give a rough outline of DG and VT; a comprehensive research overview can be found in the two volumes of the Handbook of Dependency and Valency (Ágel et al. 2003; 2006), which features altogether 121 articles in German or English. The present section is organized as follows: sections 10.1.2 and 10.1.3 introduce Tesnière's DG and VT, while section 10.1.4 outlines the central properties of a modern DG and VT. 


\subsubsection{Tesnière's structural syntax}

The founder of modern DG and VT is the Frenchman Lucien Tesnière (1893-1954) whose main work Eléments de syntaxe structurale was published posthumously (1959; 1976). The Eléments had essentially been completed in the 1940s. A short summary under the title of Esquisse d'une syntaxe structurale was published in 1953. There is a shortened translation of the 670-page Eléments into German (Tesnière 1980), but none into English. Tesnière's theory of structural syntax-only his followers named it "dependency grammar"—has the following basic features:

1. It combines a typological with a universal outlook.

2. It accounts for the infinity of theoretically producible and analyzable sentences.

3. It is autonomous.

4. It is functional.

Tesnière, who spoke more than 20 languages and used examples from more than 60 languages in the Eléments, placed great emphasis on applying his descriptive apparatus to different languages and linguistic types. He realized this typologicaluniversal outlook through a modular approach: the Éléments divide into three main parts, of which each deals with one of the basic relations of Tesnière's structural syntax: connexion, jonction, translation.

The 300-page section on connexions introduces DG and also VT as an organic part of the former. It describes the basic structure of sentences, which is not based on the binary divide into a subject (or NP) and a predicate (or VP) but on the principle of endocentric verb centricity. Endocentricity means in this context that the sentence structure unfolds from the lexical verb, that the lexical verb is the precondition for the sentence structure. ${ }^{1}$

Connexions refer to the structurally induced co-occurrence of words, dependency to the hierarchical ordering of connexions. We explain this using the example Peter read very good books, whose structure Tesnière would have represented in the following tree diagram (stemma):

(1)

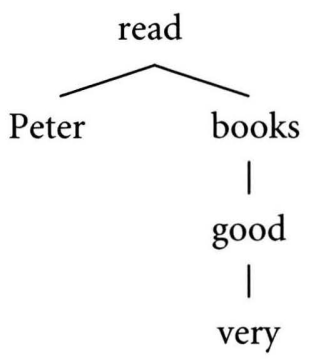

1 Although Tesnière does not use the term endocentricity, he not only investigates the respective phenomenon, but his discussion is more consistent than that of Bloomfield (1933: 195), as he assumes that in a sentence such as lat. filius amat patrem 'the son loves the father' the predicate and subject are not amat and filius respectively, but ama- and filius ...t. The verb ending ...t $t$ belongs to the verb morphologically only; syntactically it is part of the subject (Tesnière 1976: 104); cf. section 10.2.6. 
This stemma is only superficially similar to a constituency tree, it is a dependency tree. The branches are not graphical representations of part-whole relations but of connexions: seen from the top, they are government relations, seen from the bottom they are dependency relations. The element at the top, the regent, ${ }^{2}$ creates a structure for one or several dependents. The graphical design of the stemma states that the sentence is primarily about a specific act of reading rather than a specific quality of the person Peter or the object books. It states further that a person as reader, realized as subject, and something that is read, realized as direct object, are involved in the act of reading. The nouns Peter and books, structurally the direct dependents of the regent read, could be involved in different expressions referring to completely different acts or scenes (Books are exciting, I gave Peter a compact disc player). The grammatical functions of Peter and books thus vary dependent on the verbal regent, but not the other way round (verb centrality). The dependency principle can also be applied to the relations between books and good, and to those between good and very: good books refers to a specific quality of books and not to a bookish quality. By very good, we mean the grading of a quality and not a qualified grading. Thus the respective connexions must be such that books is the regent of the dependent good, and good the regent of the dependent very.

The constituency structure of the sentence can be derived from the dependency structure: the whole sentence is a verbal node (nœud verbal), consisting of the top regent (read) and two noun nodes. One of them (Peter) just consists of the nominal regent (that is a dependent of the verbal regent at the same time). The other noun node (very good books) consists of the regent books (that is also a dependent of the verbal regent) and the adjectival node very good that consists of the adjectival regent good and the adverbial dependent very.

What can-in contrast to modern dependency grammars-only partially be reconstructed, is word order. Tesnière differentiates strictly between the multidimensional structural order of sentences and their one-dimensional linear order, which is not represented in the stemma. However, the translation of linear into structural order (analysis) or of structural into linear order (production) is guided by the linguistic type of a language: Tesnière (1976: 22ff.) differentiates between centrifugal languages that linearize the structural order in a descending manner: first the regent, then the dependent (fr. cheval blanc), and centripetal languages that linearize the structural order in an ascending manner: first the dependent, then the regent (white horse). ${ }^{3}$

2 Note that a regent (or governor) in dependency grammar governs another word form in the first instance, and only indirectly a phrase. The term is thus used in a slightly different way from head in phrase structure grammar. Mel'čuk (2003: 191f.) differentiates between governor as the external governing element and head as the internal governor.

3 Tesnière's word order typology anticipates the basic idea of the word order typology of Greenberg (1963). 
However, let us return to the structural order. In our example above, all regents and dependents belong to four word categories: verb, noun, adjective, and adverb. It is not by accident that it is exactly these four. According to Tesnière, there are universally only the four lexical word classes verb, noun, adjective, and adverb that have node building power. Also universal are the dependency relations that regulate the possible constellations between the lexical classes: the verb is the top regent, it governs but cannot be governed. Nouns are direct dependents of verbs and direct regents of adjectives. Finally, adverbs are direct dependents of verbs ( He read SLOWLY), adjectives (VERY good), or adverbs (VERY slowly). This leads to the following universal dependency structure:

(2)

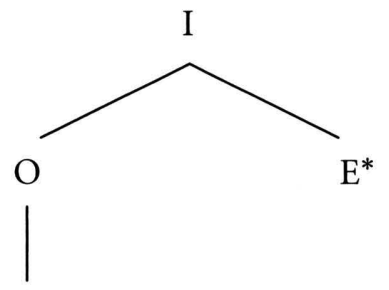

A

$\mid$

$\mathrm{E}^{*}$

Imitating the Esperanto endings, Tesnière introduces the following symbols:

$\mathrm{I}=$ verb; $\mathrm{O}=$ noun; $\mathrm{A}=$ adjective; $\mathrm{E}=$ adverb. $\mathrm{E}^{*}$ stands for a recursive relation, i.e., adverbs can also depend on adverbs. Tesnière calls a stemma that contains symbols a virtual stemma and one that contains word forms an actual stemma.

Our introductory example is simple in a literal sense since

(a) all regents and dependents are simple word forms (Peter, read, books, good, very);

(b) all regents and dependents can be attributed well-defined grammatical functions: first noun node $=$ subject $=$ Peter, $v e r b=$ predicate $=$ read, second noun node $=$ direct object $=$ very good books, adjective node $=$ first grade attribute of first degree $=$ very good, adverb node $=$ attribute of second degree $=v e r y .{ }^{4}$

But linguistic reality is far more complicated. For instance, predicates often do not just consist of a single verb form but of a verb complex (e.g., have read). Nouns can not only be specified by adjectives but also by many other elements (e.g., Laura's). And all regents and dependents can be doubled or multiplied through co-ordination (e.g., Peter and Paul). The basic structure of sentences can thus be expanded through qualitative and quantitative operations (Tesnière 1976: 80), e.g., Peter and Paul have read Laura's book:

4 Tesnière does not use the traditional functional terms predicate, subject, object, and adverbial but replaces them with the notions of central nucleus, actant, and adjunct according to his basic principle of endocentric verb centricity (see below). 
(3)

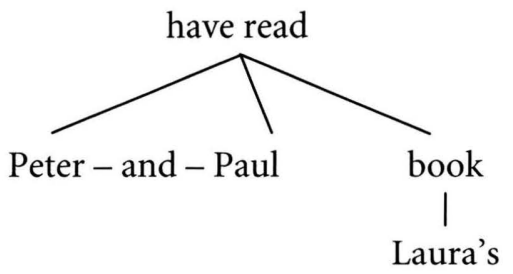

This sentence contains both simple and complex regents and dependents. Regents and dependents have both lexical and structural (grammatical) aspects. To reflect this, Tesnière calls simple and complex regents and dependents nuclei (nucléus). In the case of a simple nucleus such as read in (1) the lexical and grammatical aspects are united in one word form. Dissociated nuclei such as have read or Laura's, on the other hand, have a structural regent (have or 's) that contains mainly grammatical information, and a lexical regent (read or Laura) that contains mainly lexical information. Tesnière's DG thus contains all elements of a modern phrase structure grammar: the concept of a phrase corresponds to the concept of a node. The structural regent of a nucleus is the head, the lexical regent the core of a phrase. If the nucleus consists of just one word form, head and core coincide.

The responsibility for the qualitative expansion of the basic structure by complex nuclei lies with the basic relation of transfer (French translation), the responsibility for the quantitative expansion of the basic structure by increasing the number of (simple and complex) nuclei lies with the basic relation of junction (French jonction). While connexion (dependency) is the inter-nuclear relation of super- and subordination, junction is the inter-nuclear relation of co-ordination. Junction is a quantitative phenomenon that can be compared to addition or multiplication (Tesnière 1976: 324). It operates either with a junctive such as and (Peter and Paul) or without junctive (veni, vidi, vici).

Much more complicated is transfer. As there are only four categorial types of nuclei, whose possible dependency relations are strictly regulated, all types of complex structures have to be converted into these four categorial types. Transfer is an intra-nuclear relation that consists of two types of operations (Tesnière 1976: 364): (1) change of category, and-as a consequence of this-(2) change of function.

Our examples for transfer are the complex nuclei Laura's and have read. As the nucleus Laura's in the noun node Laura's book occurs as a dependent of the noun book, according to the universal dependency structure it has to belong to the category of adjective. Likewise, the nucleus have read must belong to the category of verb. But now consider that neither can Laura's belong to two word classes (noun and adjective) at the same time nor can a complex nucleus comprising at least two words belong to a single word class! Therefore the categories of Laura's and have read cannot be the genuine word classes of adjective and verb respectively but 
must be categories that have been created as a result of an operation. The theory of transfer describes the mechanisms and types of such category creation processes. Each transfer features a transferent (French transférende), a word the category of which is to be changed, and a translator or translating agent (French translatif) which does the grammatical work of effecting the category change. The latter can be a free, bound, or zero morpheme. The result is the translatum (French transféré), the operatively created nucleus: the clitic's changes the noun Laura into the adjectival nucleus Laura's $(\mathrm{O}>\mathrm{A})$, which as a result of the transfer is structurally dependent on and functionally attributed to the noun book. In the case of the translatum have read the verbal transferent read has-with the help of the translator in the form of the auxiliary have-been transferred from a verbal subcategory, in modern parlance a synthetic verb form, into another verbal subcategory, an analytic verb form; thus I > I (verb is transferred to verb). ${ }^{5}$

(3a)

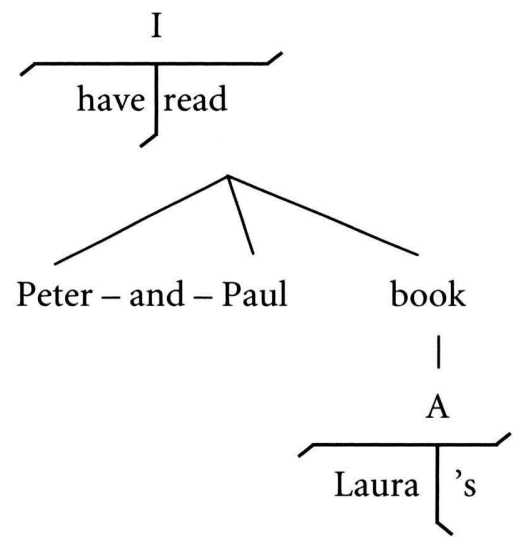

At the end of this section we would like to come back to the four basic features of Tesnière's DG. We hope to have shown in our sketch that Tesnière's DG is a

1. typological-universal theory

2. that is designed to account for the infinity of theoretically producible and analyzable sentences.

But what does the claim mean that Tesnière's structural syntax is

3. autonomous and

4. functional?

By autonomy of his syntax Tesnière means that syntax, which investigates the inner form of the sentence, is conceptually different from morphology, which investigates the outer form of the sentence (Tesnière 1976: 34). Both should thus be kept separate in description: syntax adheres to its own laws.

5 Tesnière chooses a $\mathrm{T}$ to represent transfer graphically. The translatum (or its symbol) is placed above the horizontal T-bar, transferent and translator are placed below the horizontal T-bar to the left and right of the vertical T-stroke (or the other way round). 
Syntax is autonomous since it is as clearly differentiated from semantics as it is from morphology (Tesnière 1976: 4off.). Here Tesnière anticipates the argument of the early Chomsky: he sees the best proof for the independence of syntax from semantics in semantically absurd sentences such as Vertebral silence upsets the lawful sail (le silence vertébral indispose la voile licite), which are structurally correct, possessing the same structure as meaningful sentences.

The autonomy of syntax from semantics does not mean for Tesnière that semantics is linguistically of no interest. On the contrary: meaning is the ultimate raison d'être of structure and thus the indirect subject of syntax (Tesnière 1976: 40). We have learned from connexion theory and its interplay with transfer theory that Tesnière's syntax theory is functional. The simple nuclei in the introductory example (1) allowed a direct functional interpretation of the nuclei and nodes. To ensure that a functional interpretation of complex nuclei such as (3) is guaranteed, nucleus categories created by transfer were permitted in addition to the original nucleus categories. In Tesnière's thinking, structure only exists to the extent that there are functions. Thus his structural syntax is also a functional syntax (Tesnière 1976: 39). We interpret Tesnière's functional outlook as a logical and consistent attempt to motivate the autonomous grammatical structure by establishing a theoretical link between semantics and grammatical functions.

\subsubsection{Tesnière's valency theory}

In his famous drama metaphor Tesnière (1976:102) compares the sentence to a small drama. In a similar way to a drama encompassing an action or event, actors and (temporal, spatial, etc.) circumstances, the verbal node contains a central nucleus, which, according to the universal dependency structure, can govern two types of nodes: noun nodes and adverb nodes:

(4)

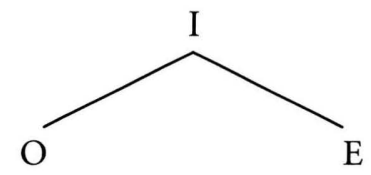

(5)

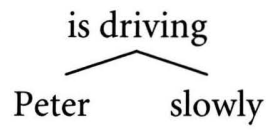

The central nucleus corresponds to the drama event: it is a (simple or transferred) verb (is driving), which in modern VT is called a verbal valency carrier. The functional equivalent of the drama actors are the actants, which are noun nodes (Peter). In modern VT actants are also called complements. The functional equivalent of the circumstances are the circumstantials (French circonstants), which are adverb nodes (slowly). In modern VT they are also called adjuncts. 
The traditional principle of the binary divide of the sentence into subject (or NP) and predicate (or VP) is thus replaced by the principle of verb centricity that establishes a functional threepartite division of the sentence into verbal valency carrier, actant, and adjunct. This functional division is secured in the categorial division into verb (equivalent), noun (equivalent), and adverb (equivalent). However, actors and circumstances, or actants and adjuncts, do not have an equal input into a drama or a sentence respectively. The constitutive elements of a drama are event and actors, the constitutive elements of a sentence the central nucleus and the actants. The circumstances in a drama or the adjuncts in a sentence, on the other hand, have the external function of framing the constitutive participants. There is only a dependency relation between $\mathrm{I}$ and $\mathrm{E}$, while between I and $\mathrm{O}$ there is a valency relation in addition to the dependency relation. Tesnière uses a famous comparison, his atom metaphor, to introduce this additional relation. According to this metaphor one can compare the verb to an "atom with a particular number of hooks that can-according to the number of hooks-attract a varying number of actants, which it keeps in its dependence. The number of hooks that a verb possesses, and consequently the number of actants that it governs, constitutes what we call the valency of a verb" (Tesnière 1976: 238). ${ }^{6}$

Valency is thus according to Tesnière the number of the potential actants of a verbal valency carrier. The actants are anchored in verb meaning. For instance, French donner or English to give demand three actants. These verbs are trivalent, notwithstanding whether apart from sentences such as Alfred donne le livre à Charles (Tesnière 1976: 107; 'Alfred gives the book to Charles') there are also sentences such as Alfred donne aux pauvres ('Alfred gives to the poor') or Alfred donne la main ('Alfred holds hands') that only realize part of the valency potential of donner (Tesnière 1976: 239).

Tesnière distinguishes between three kinds of actants (Tesnière 1976: 107ff.). All three have the dependency grammatical form in common: they are noun nodes. They also share proposition formation with the verbal nucleus. Their difference lies in their semantic relation to the verbal nucleus: the first actant, the traditional subject, is the actant that carries out an activity (in the active clause), the second actant is the actant to which an activity or action happens, and the third actant is the one to whose benefit or detriment something happens. The later concepts of deep cases, semantic or theta roles are easily recognizable in this description.

Tesnière, who, in keeping with his typological interests, runs the different formal types of actant realization of various languages past the reader, does not overlook the problem of complement-adjunct differentiation that has come to occupy

\footnotetext{
${ }^{6}$ On peut ainsi comparer le verbe à une sorte d'atome crochu susceptible d'exercer son attraction sur un nombre plus ou moins élevé d'actants, selon qu'il comporte un nombre plus ou moins élevé de crochets pour les maintenir dans sa dépendance. Le nombre de crochets que présente un verbe et par conséquent le nombre d'actants qu'il est susceptible de régir, constitue ce que nous appellerons la valence du verbe. [Bold in the original]
} 
such a central place in modern valency theory. Particularly problematic here are the prepositions. According to the universal dependency structure, they have to be translators for both nominal and adverbial translata. For example, in the sentence Alfred donne le livre à Charles à Paris the chain à Charles is a noun equivalent, while the chain à Paris is an adverb equivalent:

(6)

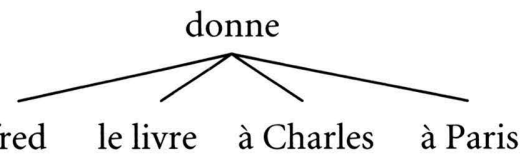

Here, valency criteria that are independent from the valency structure must decide why à Charles constitutes a transfer of $\mathrm{O}$ (Charles) $>\mathrm{O}$ (à Charles), while à Paris one of $\mathrm{O}$ (Paris) > E (à Paris):

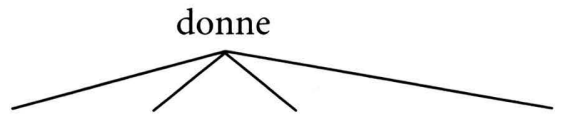

Alfred (le livre)
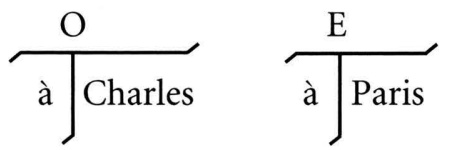

Tesnière can draw here on semantic criteria (proposition formation, semantic role) and on the fact that adjuncts are optional on principle. Also, the chain $\grave{a}$ Paris can be replaced by a single adverb (ici, là), but not the chain à Charles. Tesnière uses an additional typological argument: in case languages the chain $a$ Charles corresponds to a single noun in the dative (Tesnière 1976: 114f.).

Finally, we would like to address the question of Tesnière's relevance for modern VT and syntax:

1. Tesnière does not only demonstrate that a syntactic theory without VT is impossible, he also derives his VT from his syntactic theory in a logical and consistent fashion. Conversely he shows that valency provides an additional motivation for the universal dependency structure in that the valency relation operates on the government relation of $\mathrm{I}$ to $\mathrm{O}$.

2. In Tesnière's work, all fundamental questions of modern VTs are addressed extensively and instructively: (a) the problem of the valency carrier; (b) the problem of complement-adjunct differentiation; (c) the distinction between valency and its obligatory or optional realization; (d) the problem of structural valency realization, i.e., the question to what extent the structures of individual languages influence the forms and types of possible actants.

3. As languages like English or Chinese mark actants not just morphologically or prepositionally but also positionally, the problem of structural valency realization concerns the fundamental question of DG of whether Tesnière's rigid separation between structural and linear order should be maintained. Among the 
modern dependency grammars, there are projective grammars which preserve word order (cf. Eroms and Heringer 2003).

\subsubsection{Fundamental traits of dependency grammar and valency theory}

All grammatical theories assume that multidimensional structures are hidden behind the temporal sequences of spoken signs. Their architecture cannot be simply read off the spoken data, nor does a surface-oriented morphological description capture it: a syntactic theory must thus provide own categories and relations to describe this architecture. Tesnière's autonomy principle is based on the categorial independence of the syntactic theory component. His dependency grammar reflects semantic (and pragmatic) differences to the extent that they can be mapped onto syntactic categories, but syntax is not reduced to the expression of semantics (and pragmatics). This non-reduceability of syntax follows not only from the impossibility of projecting every semantic (and pragmatic) difference and opposition onto syntactic categories but also from the mechanisms of language change and grammaticalization. DG and especially VT put great emphasis not just on the typological but also on the linguistic-historical adequacy of theory formation. The theory should not just be applicable to a few modern literary languages but to any language at any historical stage.

The linguistic forms underdetermine the sentence structure. This is one of the reasons why this handbook presents different grammatical theories, and not concepts of a unified theory of grammar. A second reason is that there is no consensus on which concepts are the basic ones for describing linguistic structure (e.g., dependency or constituency) or even which concepts are necessary (e.g., functional notions such as subject and/or formal ones such as NP). Another question is how complex or abstract should the structural descriptions be. The crucial point is that a methodologically controlled link between theory and empirical data must be guaranteed. This challenge is closely connected to the description and explanation of grammatical constructions: How should a syntactic theory deal with these? Should it take (a) a projectionist perspective (from the elements to the whole), or (b) a constructionist perspective (the other way round)?

DG is fundamentally a projectionist theory, which describes structure starting from the individual lexemes: DG is a word grammar. Individual lexemes have a combinational potential or tolerance, which creates or does not prevent or excludes structures. DG is thus, in this respect like HPSG, not a "slot theory" (Feilke 2004: 54 ), in which the lexicon merely provides fillers for lexically independent syntactic structures. An essential part of grammar is in the lexicon: in the potential of lexemes for connexion, junction, transfer, and valency. This word grammar perspective of DG has a number of consequences: 
1. The combinational potential of individual lexemes is founded on their meaning and their syntactic potential. But how does the autonomy principle of DG interact with this lexical foundation? In most cases the dependency branches can be directly semantically interpreted. But no identity or one-to-one correspondence between the structural and the semantic level is assumed, as for instance in categorial grammar and in the basic outlook of functional approaches. As a structural theory, DG assumes an independent structural level. Only with this assumption is it possible to ascertain the degree of correspondence between syntactic and semantic structures (Welke 1988; Helbig 1992). The word-grammatical perspective, on the other hand, ensures that the degree of syntactic independence is as small as possible, and that syntactic structures are motivated semantically. DG shares this search for semantic motivation with categorial grammar and functional theories, in spite of the differences outlined above.

2. DG's lexical perspective, i.e., its taking individual lexemes as the point of departure, is compatible with its interpretation as either a reception or a production grammar. The view of DG as a reception grammar is possible because a recipient's knowledge of the syntactic-semantic potential of individual lexemes, supported by framing and priming, leads to reliable analyses (Heringer 1984; 1985; Gansel 2003). The view of DG as a production grammar is possible because the production of utterances depends on the choice of individual lexemes both on the basis of communicative intention and-trivially but crucially-on the basis of the syntactic-semantic opportunities and restrictions that have been conventionalized in the vocabulary of an individual language. For instance, the English verb lie can hardly be combined with a that-clause expressing the content of a lie (Fischer 1997: 241), while a corresponding construction is acceptable in Hungarian: ${ }^{7}$

(7) English ?He lied that he was poor.

(8) Hungarian Azt hazudta, hogy szegény.

that $t_{\text {Pron.Acc }}$ lied-he that Conj $_{\text {poor }}$

3. DG is a data-friendly syntactic theory in the sense that it is not restricted to the representation of a few abstract structures but aims at the description of a considerable sample of natural language. DG is also a data-friendly syntactic theory in the sense that it aims at a level of abstraction that still permits a methodologically controlled link to the data.

4. The word-grammatical perspective makes DG particularly suitable for natural language processing (parsing, generation, translation).

5. The relative simplicity of dependency grammar representations makes DG suitable for didactic purposes. DG representations are used in a number of learner grammars and German as a foreign language material.

${ }^{7}$ Acc $=$ accusative, Conj $=$ conjunction, Pron $=$ pronoun. 
From the lexical perspective it follows that the central question of DG is the justification of dependencies, i.e., the motivation of connexions between words and their hierarchical ordering. In modern DG both are-to a greater extent than by Tesnière-justified using valency relations (e.g., Engel 1994). Nevertheless, both are not identical: DG is a general syntactic theory which, according to Welke (1995), can be founded on three types of dependency relations: government, endocentricity, and subcategorization (cf. 10.3.3). VT is a partial theory, which is concerned with the combinational potential and realization of relational signs (verbs, nouns, and adjectives). VT cannot but be integrated into other syntactic theories, its integration is essential: no grammar with comprehensive descriptive and explanatory ambition can do without a valency component.

We conclude this section with the provocative question: "Is DG the best of the theories presented in this handbook?" In spite of the many advantages that DG has over its competitors and that are demonstrated in this chapter, a positive answer would be premature. Neither DG nor other theories can presently lay claim to the title of best theory. As Jacobs (2008) shows convincingly, there are a number of central, i.e., not peripheral, grammatical phenomena that can only be described adequately from a constructionist perspective. A (different) number of equally central grammatical phenomena, on the other hand, require a projectionist perspective. A convergence of projectionist and constructionist grammatical models is definitely required (cf. 10.2.5, 10.2.7, and 10.3.4). The future of DG-as that of other theories-thus depends in great measure on whether the DG framework (or that of another theory) allows the development of a theoretical format that achieves the integration of projectionist and constructionist components-not just as ad hoc stipulation but in an empirically and methodologically sound way.

\subsection{VALENCY THEORY}

\subsubsection{Contextualization}

Human language does not construct its messages holistically but in predicateargument structures: events are perceived as consisting of things and relationships between these things. Nouns refer to entities, among which a verb as a relational expression creates a link. The referents of verbs are more abstract and can only be perceived indirectly: for instance, you can only point at them by pointing at one or several objects (cf. Leiss 2002).

All languages possess linguistic means to create predicate-argument structures (proposition formation). Valency theory (VT) investigates this essential core of 
human language, both as a universal and as a particular shaped by the characteristics of individual languages.

Modern VT derives from Tesnière, but the valency idea is much older. K. Bühler (1982 [1934]) and J. W. Meiner (1971 [1781]), for instance, are seen as direct precursors, but valency-like concepts have been identified in antiquity, in the Arabic grammatical tradition and among the medieval modists. Both Tesnière and his followers see themselves in the tradition of European structuralism, but the fact that the development of post-Tesnière VT was initially centered on central and eastern Europe is not just a historical accident but can be explained by the obvious application of VT to case languages. By now there exists valency research on a multitude of languages, especially on Romance languages and on English. The number of publications on valency is estimated to comprise around 3,000 titles.

The development of VT did not take place in isolation from the predominantly Anglo-Saxon linguistic approaches that dominated the linguistic scene in the second half of the twentieth century: the semantic feature analysis by J. Katz and J. Fodor (1963), the deep case (semantic role), later scene concepts of C. Fillmore $(1968 ; 1977 a)$, the general turn to semantic-pragmatic approaches in the 1970 (generative semantics, functional grammars) should be mentioned as well as categorial grammar, the typological opening up of VT in the last decade, and the recent discussion of concepts from construction grammar (Willems and Coene 2006). Valency theoreticians see related concerns realized in (other) word grammars (Hudson 2006; Starosta 1988) and in functional approaches (Halliday 1994; Dik 1978; Givón 1995).

VT's openness to concepts developed outside its framework is hardly reciprocated by other approaches (but cf. Huddleston and Pullum 2002). However, grammars today are highly likely to possess a valency-type component (of variable analytical depth), usually under a different name (e.g., subcategorization). These valency-type components have moved closer to VT. This is particularly obvious in the X-bar template of generative grammar. Recent modeling of first language learning, which emphasizes the lexeme-specificity of the initial acquisition of syntactic functions (Verb Island hypothesis, Tomasello 2003: 117), supports the word grammar outlook of VT.

Four basic questions of VT can be identified (Ágel 2000: 115ff.):

1. What counts as a valency carrier?

2. What is the complementation potential of a valency carrier?

3. Which forms and types of valency realization exist in different languages?

4. How is valency realized in texts?

In section 10.2.2 we will discuss extensions of the valency concept after Tesnière, followed by discussion of questions 1 and 2 in sections 10.2.3-10.2.5. Section 10.2.6 deals with the typological question 3 . For reasons of space, question 4 will only be 
marginally touched upon. And in sections 10.2 .7 and 10.2.8 we will briefly address valency change and applied valency research.

\subsubsection{Extensions of the valency concept after Tesnière}

\subsubsection{Fundamentals}

VT is based on the very simple idea or observation that words pre-determine their syntactic and semantic environment:

(9) The man is washing his shirt.

The verb wash creates two slots that require a particular categorial filling in a particular function. The verb allocates the function of a washer (agent) to the first slot and the function of a washed (patient) to the second slot: the washing scene is thus seen from the perspective of the washer, the verb wash opens a perspective on the washing scene (Welke 2005).

The functional requirement of the verb largely determines the categorial filling of the slots. In literal usage, only entities with the feature "material" can wash or be washed. And only an entity that can carry out an activity can wash. Correspondingly, the categorial filling of the slots is restricted: subject and object must be realized as NP (noun nodes), not, for instance, as PP (prepositional nodes).

The semantic restrictions here are a direct reflex of our knowledge of washing events. But lexemes are arbitrary in the sense that reality does not force us to form a particular linguistic notion. Reference to washing events would have been possible using a generic verb and an adverbial phrase (clean with water). Also, the exact delimitation of what we can refer to using the verb wash is not prescribed by reality. In English, cleaning the floor with water may be referred to as wash(ing) the floor, but the German equivalent of wash is usually not used in this context, but the verb wischen ('wipe') is. If one also takes metaphorical usage into consideration, English wash is used less widely than German waschen (Geld waschen vs. launder money, cf. Feilke 1998: 75).

Verbs are not just combined with complements but also with adjuncts:

(10) The man is washing his shirt in the kitchen.

The PP in the kitchen situates the washing event. We know that all physical events take place at a certain place and time, but this is world knowledge and not a requirement of the lexeme wash. Sentence (9) above represents a complete linguistic realization of the scenario ${ }^{8}$ created by the verb wash: no local or temporal information is assumed or must be supplied. This is different in

\footnotetext{
8 The term scenario refers to linguistically created event types or events, not to languageindependent states of affair in reality.
} 
(11) He put the shirt in the kitchen.

(12) *He put the shirt.

In (11) the adverbial of location (in the kitchen) is constitutive for the puttingscenario: put opens a slot for an adverbial of location. No minimal putting-scenario can be realized without this slot being filled (cf. 12).

We have now prepared the ground for offering a valency description of the combinatorial potential of the verb wash:

(13) wash <AGENT: material (living being, institution, machine) $\rightarrow$ subject: NP; PATIENT: material $\rightarrow$ direct object: $\mathrm{NP}>$

VT emphasizes that the different syntactic functions such as subject, direct object, obligatory adverbial, etc. have something in common: to fill verb slots. Therefore, including the subject, they are subsumed under the notion of complement, though they are very different in other respects.

\subsubsection{Formal marking of valency}

Tesnière restricted valency to the number of complements. In the understanding of modern VT, verbs determine both the number and kind of slots (e.g., Helbig 1992). The most tangible expression of valency exists perhaps in the marking of complements using case: ${ }^{9}$

(14) German Der ${ }_{\text {Nom }}$ Hund folgt dem Dat $_{\text {Mann. }}$

'The dog follows the man.'

In German the follower must be realized in the nominative, the followed in the dative, with cases mainly marked on the determiner. Permutation of the unmarked order "subject before dative object" changes the information structure, but not the event expressed:

(14a) Dem Mann folgt der Hund.

'The dog follows the man.'

Positional marking of nominal complements often occurs in languages lacking case. For instance, the unmarked order of nominal complements in English was grammaticalized in parallel to the loss of case:

(15) The dog follows the man.

(16) The man follows the dog.

The follower occupies the preverbal, the followed the postverbal position. Contrary to German, an exchange of positions (16) changes the stated event.

An additional formal marker is provided by verb-specific prepositions, which are often considered as case equivalents:

\footnotetext{
${ }^{9}$ Nom $=$ nominative, Acc $=$ accusative, Gen $=$ genitive, Dat $=$ dative.
} 
(17) She insists on $/{ }^{*}$ under $/{ }^{*}$ in $/ *$ for treating everybody with courtesy.

Two important questions arise from the formal marking of complements:

1. To what extent is formal marking unequivocal?

2. Does formal marking just differentiate between complements, or does it also carry semantic information?

Neither position nor morphology offer unequivocal marking: although positions as such are unambiguously defined (before $\mathrm{X}$, after $\mathrm{X}$ ), they are not restricted to marking just one function. The definition of English nominal complements therefore has to be based on unmarked or canonical positions (cf. Huddleston and Pullum 2002: 225f.). Morphological marking systems have a tendency toward form syncretism (often subject-direct object; cf. Plank 1984). On the other hand, the number of differentiations that can be achieved in case systems is potentially less restricted than the number of grammaticalized positions.

It would be uneconomical if formal marking were merely to serve complement differentiation, with the mapping of formal markers onto semantic roles happening in an idiosyncratic fashion, e.g., an agent being marked unsystematically by a variety of positions, cases or prepositions. In fact, the mapping follows regularities that are both based on competing principles and are sensitive to the presence of other complements.

\subsubsection{Adjectival and nominal valency}

Not only verbs have valency but also adjectives (proud of his children, keen on debate) and nouns (protection from disease, Peter's sister). Individual adjectives need additional information to express their quality, e.g., of whom or what somebody is proud. Likewise, the constitution of entities sometimes requires further protagonists, e.g., protection from something. Although adjectival and nominal valency is frequently deverbal (bore $s b d y \rightarrow$ boring for $s b d y$; protect sbdy from sthg $\rightarrow$ protection of sbdy from sthg), this is not always the case, as the initial examples show. There are currently three competing approaches in existence in the theory of nominal valency. Only one of them, the so-called nominalization approach, assumes that there is no genuine nominal valency but that the nominal valency structure is inherited from the verb. This approach, which is comparable to $\mathrm{X}$-bar theory, is seen as refuted in valency theory (Teubert 2003).

\subsubsection{Valency potential I: Complements and adjuncts}

\subsubsection{Valency as a unified phenomenon}

For a long time, it had been assumed in VT that valency was a unified phenomenon. Therefore, the delimitation of complements from adjuncts (c/a-delimitation) was 
seen as fundamental to the development of VT. Considerable time was invested into attempts to operationalize c/a-delimitation (which seems intuitively very clear), but no consensus was achieved. The problem of c/a-delimitation derives from a number of phenomena:

1. Complements can be optional, thus optionality is not a sufficient criterion for adjunct status:

(18) She is reading a book.

(19) She is reading.

The direct object $a$ book is "truly optional" (indefinite deletion) since the patient of the reading process does not have to be contextually given for (19) to be understood.

2. As a phrase can function both as a complement and as an adjunct, there is no straightforward formal criterion for c/a-delimitation. The same chain can be $\mathrm{c}$ or $\mathrm{a}$, depending on the verb (see in the kitchen in (10) and (11) above) or depending on the scenario that an utterance is intended or interpreted to realize:

(20) She meditated yesterday evening.

(20) is syntactically ambiguous, since yesterday evening can be interpreted as c (direct object) or as a (temporal adverbial).

Solutions to the puzzle of c/a-delimitation (with respective tests) were attempted on a morphosyntactic (complements are formally determined by the verb; Eisenberg 2006: 33ff.), distributional (complements are specific to subclasses of verbs; Engel 1988), and semantic basis (verb and complements constitute a minimal proposition; Fischer 1999), but none proved wholly satisfactory. Nor did the construal of the c/a-transition as gradual or scalar (e.g., Heringer 1984 and 1985; Somers 1987) seem to adequately reflect the nature of the c/a-difference.

\subsubsection{Multidimensional valency models}

This research situation was given a surprising twist by Jacobs (1994). He claimed that there were seven (later five) valid operationalizations of the valency concept, to which mutually independent relations corresponded: valency was not a unified phenomenon at all but a cover term for these individual valency relations, which, though independent, prototypically occur together. Jacobs's intervention resulted in multidimensional (or modular) valency models, which provide the dominating paradigm of VT today (see also Jacobs 2003). The following outline of a multidimensional model is based on Zifonun et al. (1997: 1,026ff.), but adds the relation "position", thus listing the valency relations needed for the description of English and German. The relations can only be presented but not discussed in any detail: 
a) Form relations

(i) Obligatoriness:

He devours a hotdog. ${ }^{*}$ He devours.

(ii) Form determination:

(iii) Constancy:

They can see him $/{ }^{*}$ he.

(iv) Case transfer:

She insists on $/{ }^{*}$ in $/{ }^{*}$ under $/{ }^{*}$ for meeting us.

German Sie besteht auf einem Dat $/{ }^{*}$ ein Acc $_{\text {Treffen. }}$

'She insists on a meeting.'

German Sie freut sich auf ein Acc $^{*}{ }^{*}$ einem ${ }_{\text {Dat }}$ Treffen.

'She is looking forward to a meeting'.

(v) Position:

He devours a hotdog. *A hotdog devours he.

The verb determines whether and under which conditions a complement can be omitted (i), and which form features it has, i.e., in which case it has to occur (here: the DO must occur in the objective case) (ii), which preposition provides the link to the object (iii), and, for constant or verb-specific prepositions that can govern different cases, which case is chosen (iv): the German preposition auf governs either the accusative or the dative. The choice in (iv) depends on the respective governing verb. Position (v) plays a greater role in a language such as English (cf. 10.2.2.2) than in languages with flexible complement order (e.g., Russian). The English positions are allocated by the verb as a category (subject) or by the subcategories of transitive (direct object) and ditransitive verb (indirect and direct object). Accordingly, English adjuncts possess greater positional variability (see Huddleston and Pullum 2002: 225f.).

b) Meaning relations

(vi) Proposition formation: They arrive at home.

(vii) Perspective: The car costs $\$ 3,000$. Cf. She buys a car for $\$ 3,000$.

(viii) Synsemantic coding: He spies on her. Cf. The fly is on her.

To create an arrival event, it is necessary to refer to an entity that arrives and to a place where it arrives (vi). The verb cost puts the price more strongly "into perspective" than the verb buy does (vii). The semantic relation between the verb spy and the object of spy is not created by the spatial meaning of on but depends on the verb (viii). The relation between the fly and the person, however, is captured by the meaning of $o n$.

The more relations can be attributed to a phrase, the stronger is its claim to complement status. Prototypical complements and adjuncts show convergence of the form and meaning relations. There are implications between the relations. For instance, proposition formation implies that the respective phrase is strongly or weakly in perspective.

All the relations show variability across languages. As we have shown above, the form relation position is important in English, while the role of form determination has been much diminished and case transfer has ceased to play a role at all. These relations are not equally important in determining the c/a-status of a phrase. Proposition formation, if suitably defined and not restricted to arguments (cf. 10.2.3.3), 
is at the core of valency as a universal phenomenon and decides the c/a-status of phrases (nodes) in most cases (cf. Fischer 1999). Form relations concern the realization of valency in individual languages (cf. 10.2.6). The other two meaning relations (perspective, synsemantic coding) are a function of the form relations, which in their turn are-at least up to a point-informed by the meaning of the verb.

\subsubsection{Complements and arguments}

Although valency is grounded in predicate-argument structures (and thus is fundamentally a semantic-pragmatic phenomenon), it cannot be equated with these structures. Not all arguments are realized as complements, and not all complements are arguments. Adjectives, for instance, have an argument slot that is not filled by a complement inside the adjective phrase but by the noun to which the adjective refers (a proud girl, she is proud of her children). Semantic valency slots can also be realized as part of lexical meaning or be blocked from realization: verbs such as hammer, nail have incorporated the instrument into the verb. A number of German prefix verbs prevent realization of the patient (e.g., zuschlagen: Er schlägt zu. 'He hits at somebody or something' vs. *Er schlägt ihn zu. 'He hits him'), although a patient is assumed.

VT thus cannot be reduced to the predicate calculus, though both take the propositions of linguistic utterances as their point of departure.

\subsubsection{Valency potential II: The valency carrier}

\subsubsection{Identification of the valency carrier}

The determination of valency (or the valency relations) of $\mathrm{X}$ assumes that we have identified X (the valency carrier):

(21) The woman is washing her blouse.

What is the relational sign in (21)? The system element wash or an instance (token) of the word wash in a text (e.g., washing) or a verb form of wash (e.g., is washing)? Do we assume just one valency of wash or different valencies of different partial paradigms (e.g., active, passive) or even of individual word forms? Does a polysemous verb such as realize (cf. 10.2.4.2) have one valency carrier or several? Then there is the question of how to incorporate idiomatic expressions into VT. Is the valency carrier of

\section{(22) He kicked the (*big) bucket/*frying pan. ('died')}

the unanalyzed monovalent valency carrier kick the bucket? Or should an "internal valency", derived from the verb kick, be assumed inside the idiomatic expression?

Realistically, idiomaticity cannot be excluded from VT: not only is the free combinability of elements rather the exception but also idiomatic expressions do possess limited variability (see Ágel 2004): 
(23) She kicked up a (big/small) fuss/row/stir/stink/?an argument/* a problem.

Further delimitation problems arise from prepositional and particle verbs: does after in look after somebody belong to the valency carrier or to the complement? (Does the verb look after govern a direct object or the verb look a prepositional object? Cf. Quirk et al. 1985, Emons 1974). Is on in put on a dress part of the valency carrier or an adverbial complement?

\subsubsection{The problem of readings}

In addition to the external identity problem, the delimitation of the valency carrier of verbal idioms and particle verbs, there exists an internal identity problem. For most verbs the simple equation of "one valency carrier = one verb" does not work as verbs tend to have different readings (cf. the Variantenproblem in Ágel 2000):

(24) She realized her plan. ['make real']

(25) She realized that her purse was missing. ['notice', 'become aware of a reality']

Although the readings share a meaning that may be identified as "real" (if suitable paraphrases of the readings are chosen), it is not possible to derive from this meaning element and the function of the word formation suffix -ize that the verb realize can both be used as an action verb with an agent and patient (24) and, more frequently in current usage, as a verb of perception with an experiencer and a stimulus (25). Accordingly, it will hardly be possible to subsume both readings under one paraphrase. Nevertheless, the two readings are not just homonyms but readings of one verb that are semantically connected.

Given our findings, how can it be maintained that the lexeme realize determines number and kind of its complements? The lexeme does not seem to achieve this. At least the kind of complements can only be derived if the meanings of the individual readings serve as the starting point. If one starts from the lexeme realize, it is the direct object that determines the verb: if it refers to a fact (e.g. "that her purse was missing" in 25), the reading "notice" is chosen.

But the problem of readings reaches even deeper. How many readings does the verb realize have? The Longman Dictionary of Contemporary English indicates five, Collins Dictionary of the English Language seven, without there being a straightforward mapping between the two sets of readings.

We conclude that verbs do not only determine their environment but that they are also determined by it: the meaning of verbs is often abstract as verbs have a number of usages that are interconnected through family resemblances. Thus their interpretation needs input from both the linguistic context and the situation. This explains the strong variability of verbs across languages, i.e., cross-linguistic matches are between verb readings rather than verbs, and the (unmarked) sentencefinal position in almost half of the world's languages. This preference for positioning the verb after the complements can only be reconciled with the VT claim 
to verb centrality, i.e., the view that the verb determines the construction, if the problem of readings is considered. The relationship between verb and complements thus should be seen as a dialectic or two-stage process, in particular in reception: 1. determination of the verb (choice of verb reading); 2 . determination of the complements by a verb reading.

The relationship between inherent lexical meaning, combinational meaning, and readings is currently very much discussed in VT, also with reference to construction grammar (see Willems and Coene 2006; Coene 2006).

\subsubsection{Valency modification and alteration}

\subsubsection{Basic valency and valency modification}

For a verb such as wash, it can be convincingly demonstrated that its lexical meaning determines its valency. In 10.2.2.1 we have shown that a divalent realization derives from the meaning of wash. We consider this realization as the basic valency of wash (cf. Welke 1988). From here, a number of systematic valency modifications (valency reductions and increases) can be expected: medial usage that is often not formally marked in English (She is washing 'washing herself'), secondary subjectivization of the patient (These shirts wash well), resultative usage (He is washing his clothes to shreds), instrumental usage (She is washing the car with a hose pipe), benefactive usage (She is washing the car for her) and, in suitable contexts, the indefinite usage (He is washing 'doing the washing'). We cannot demonstrate this in detail here, but both the valency potential and the valency realizations of wash can be derived from its inherent meaning with the help of world knowledge, the interplay between the degree of agentivity of the protagonists and the case hierarchy-this interplay determines the mapping of arguments onto complements-and syntactic rules of valency modification (cf. 10.2.5.2; cf. the Alternantenproblem in Ágel 2000).

\subsubsection{Valency alteration}

Valency modifications such as She forgot (from: She forgot to wash the car) (reduction) and She is washing the car with a sponge (increase) can be addressed as scenario conserving valency modifications (Fischer 2003). In both cases, the basic scenarios, the forgetting and the washing scenarios, have been preserved in spite of a valency modification. However, the valency increase in (27) changes the scenario of (26):

(26) German Der Hund ist weggelaufen.

'The dog has run away.'

(27) German Ihm ist der Hund weggelaufen.

$\mathrm{Him}_{\text {Dat }}$ is the dog away-run 
'The dog has run away while in his care.'/

'It's his responsibility that the dog has run away.'

While a running-away scenario is construed in (26), the addition of a dative complement results in a secondary "responsibility scenario" being created, the responsibility relating to the primary running-away scenario (27). The valency carrier weglaufen does not possess a slot for a dative complement: the responsibility scenario is imposed on the verb.

The concept of a scenario altering valency modification or valency alteration (Fischer 2003) defuses various problems of VT: the problem of readings, the problem of verb form valency, the problem of c/a-delimitation, the problem of textual valency realization. It is a theory component which enables the integration of a considerable number of constructionist phenomena into a projectionist theory (cf. the problem outline in 10.1.4).

\subsubsection{Structural valency realization}

Tesnière already saw clearly that the binary differentiation between valency as a potential of a lexeme and valency as textual-situational realization of this potential (Tesnière's valence libre) does not provide a sufficient foundation for creating a typologically adequate VT (cf. footnote 1 ). Rather, the realization of the valency potential is also determined by general dependency-structural properties of languages (or varieties). For example, in the Romance languages (with the exception of French) pronominal subjects are not realized in the unmarked case:

Italian Cerco una casa.
Look-for-1.pers.Sg.Pres. a home
'I am looking for accommodation.'

Is the Italian subject partially optional? We argue that the question is asked on the wrong level. A realization of the pronominal subject in Italian cannot be equated with the realization of a pronominal subject in, for instance, English, as the Italian realization is emphatic:

(29) Italian Io cerco una casa.

'I am looking for accommodation'/

'It is me who is looking for accommodation.'

The non-realization of pronominal subjects in Romance languages is not a question of register, text, context, or situation. We are rather dealing here with a different type of valency realization, the structural valency realization. (28) is not subjectless: the verb form cerco functions as valency carrier and first complement in one! This complement realization inside the verb form has, since Pasierbsky (1981), been addressed as "micro valency" (cf. the pro-drop parameter of generative grammar). 
Structurally, the "standard valency model" features two different forms of complement realization: first, as an inflectional morpheme that is incorporated into the valency carrier (micro valency) and, second, as a separate phrase (macro valency). Micro valency is not restricted to the subject (see László 1988):

(30) Hungarian Eszi.

\section{'He/she/it eats it.'}

Hungarian Eszi features micro-realizations of both subject and direct object, but no macro-realization.

It is tempting to assume that pronominal micro valency is the cause of the lack of pronominal macro valency in the unmarked case, but this explanation is problematic on two counts: (1) there are languages with micro-realization of the subject that also require its pronominal macro-realization (German, Islandic); (2) there are languages without micro-realizations of the first and second complement that allow pronominal non-realization of both these complements (Chinese, Korean, Japanese).

The examples show that dependency relations between micro- and macro-level can be different for individual complements and languages. ${ }^{10}$ They can thus be used for a typological classification.

The concept of micro valency has been extended beyond subject and objects to adverbial complements (Ágel 2000: 138ff.):

(31) German Sie hat das Rad an den Zaun gelehnt.

'She leaned/stood/put the bike against the fence.'

(32) German Sie hat das Rad angelehnt.

'She leaned/stood/put the bike (against sthg).'

(33) German Sie hat das Rad an den Zaun angelehnt.

'She leaned/stood/put the bike against the fence.'

(34) German Sie hat das Rad daran / an ihn angelehnt.

'She leaned/stood/put the bike against it.'

The valency carrier in (31), lehnen, requires an adverbial complement. For (32), it is traditionally assumed that the particle verb anlehnen is the valency carrier, which can optionally be combined with an adverbial complement (32 vs. 33). According to Ágel (2000: 138ff.), however, lehnen is also the valency carrier in (32)-(34): its

10 Macro-realizations of complements can be seen as dependent on their micro-realizations. In Eroms's (2000) dependency grammar, the German macro-subject does not depend on the lexical verb but on the inflectional person/number-morpheme, even if this is realized on an auxiliary verb. This looks only superficially like the dependency equivalent of the inflection phrase of generative grammar: in languages without micro valency (e.g., Chinese), all complements are dependent on the lexical verb. In languages with micro-realizations of both subject and object (e.g., some Hungarian structures), the macro-subject and -object are both dependent on their respective micro-realizations, without assuming a hierarchy. 
adverbial complement does not have to receive a macro-realization (32), if it is attached to the valency carrier in the form of the (separable) particle an (cf. head movement, Nichols 1986). Particle and valency carrier combine to create the verb form, i.e., the adverbial complement is incorporated into the verb. The case is, however, not completely parallel to the micro-realizations in (28) and (30) since the pronominal macro-realizations daran or an ihn in (34) are not necessarily emphatic (cf. Fischer 2003: 49f.). More strongly head-marking languages such as the North American languages feature micro-realization of a number of complements, including adverbial complements and adjuncts (cf. Nichols 1986).

\subsubsection{Valency change}

As was indicated in 10.1.4, VT does not just place great value on the typological but also on the historical adequacy of theory formation. Historical adequacy is stipulated in the principle of viability (Ágel 2001). According to this principle, any linguistic description or explanation of a phenomenon must be in agreement with the linguistic description or explanation of the history of the phenomenon in question. The theoretical apparatus should ideally be such that it can deal with (a) any stage in the development of a language, and (b) allows the integration of synchronic descriptions of historical stages of the same language into a diachronic model that has the current state of a language as its natural end point.

Since the second half of the 1970s, considerable time and effort have been invested into researching the valency arrangements of historical linguistic stages (in particular those of German, but also of other languages; cf. chapter XII in Ágel et al. (2006)). Less satisfactory is the development of diachronic modeling of valency change (summaries in Heringer 2006 and Korhonen 2006). Considerable empirical, methodological, and theoretical problems have been encountered in the attempt at applying modern concepts such as the c/a-delimitation or the identification of the valency carrier to older language stages (Habermann 2007). Habermann diagnoses, in comparison with New High German, a much stronger interaction of verb valency and verb meaning with verb-independent constructions and textualpragmatic factors in Old and Middle High German. Also, lexical meanings have become demonstrably better defined in the history of German (Reichmann 1988: 171). These two findings allow the preliminary conclusion that older language stages have to be described using a more constructionist and less projectionist approach than is suitable for modern literal languages. A particular challenge for a viable theory formation consists in modeling a historically unstable amalgam of grammatical phenomena that require either a constructionist or a projectionist description with a stable architecture of constructionist and projectionist theory components. 


\subsubsection{Applied valency research}

\subsubsection{Valency lexicography}

Theory formation in VT was to a considerable degree motivated by the needs of descriptive work, i.e., of lexicographic and contrastive projects. Representative examples of valency lexicography include the pioneering Wörterbuch zur Valenz und Distribution (Helbig and Schenkel 1969; 1991) and the Valenz. Wörterbuch deutscher Verben (Schumacher et al. 2004). Although German is the language that has been covered most comprehensively by valency lexicography, there are valency dictionaries for other languages, e.g., A Valency Dictionary of English: A Corpus-based Analysis of the Complementation Patterns of English Verbs, Nouns and Adjectives (Herbst et al. 2004).

\subsubsection{Contrastive valency}

A number of contrastive valency dictionaries and grammars have been compiled. We can only name a few here: Valenzlexikon deutsch-italienisch (Bianco 1996), Kontrastive Grammatik deutsch-serbokroatisch (Engel and Mrazović 1986), Kontrastive Grammatik deutsch-rumänisch (Engel et al. 1993), and Deutsch-polnische kontrastive Grammatik (Engel 1999). Three languages are constrasted in the online dictionary The Contragram Dutch-French-English Contrastive Verb Valency Dictionary (Colleman et al. 2004). For information on English-German contrastive verb valency, also from a typological perspective, see Hawkins (1986), Durrell (2002), Fischer (1997; 2007).

\subsection{DEPENDENCY GRAMMAR}

\subsubsection{Contextualization}

The dependency notion has a long tradition: in the West it exists implicitly in Antiquity, explicitly as determinatio (Boethius) since the Sixth century AD, as dependentia since the thirteenth century AD. The historical dependency concepts are significantly different from those of structural linguistics. We are only going to discuss the latter here. In 10.3.2, properties of a "pure" DG are introduced. 10.3.3 presents customary operationalizations of the dependency concept and also addresses problems of the notion of regent (head). The chapter concludes with a comparison of the two fundamental syntactic principles of dependency and constituency. 


\subsubsection{Dependency principles}

Dependency is the second means of syntactic representation alongside constituency. A third one has not been developed (Mel'čuk 1988: 13). In practice, grammars use both, but either dependency or constituency will be favored as the more fundamental means of representation. We will now introduce basic principles of a pure DG, which define the features of a dependency representation of a sentence:

1. The elements are words (or morphemes), or more precisely word forms (or morphs).

2. Exactly one element is independent (top element of the dependency tree).

3. All other elements are dependent on an element.

4. No element is dependent on itself (no reflexivity).

5. No element is dependent on more than one other element (only downward branching).

6. If element $\mathrm{A}$ is dependent on element $\mathrm{B}$, and element $\mathrm{B}$ dependent on element $\mathrm{C}$, then element $\mathrm{A}$ is indirectly dependent on element $\mathrm{C}$ (transitivity).

The result of these basic principles is an actual stemma such as:

(35)

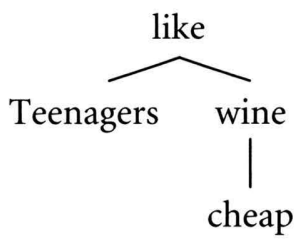

Only terminal elements occur in a dependency tree, no nodes or phrases. Each element only occurs once: the whole tree corresponds to the sentence. All elements are connected to one tree, dependency is passed down the branches. For instance, cheap is not only dependent on wine but also indirectly on like (but not on teenagers, as this word, though graphically higher than cheap, is not on the same branch). The basic principles impose considerable restrictions on pure dependency representations. They can only be maintained for partial descriptions of natural languages. For instance, the basic principle 5 is violated in the representation of the junction, for which Tesnière (1976: 340) himself used multiple regents.

Dependency grammars are word grammars: the properties of a word that are responsible for the dependency structure are detailed in the lexicon, for instance meaning, word class, valency and other distributional restrictions. This lexical information can selectively be used to label the nuclei in a more abstract fashion (cf. Engel 1994; Heringer 1996):

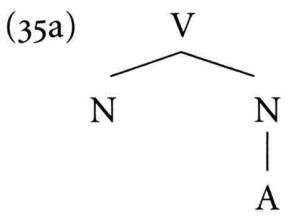


The virtual stemma (35a) also represents sentences that are structurally related to (35) such as Children eat healthy food. Both representations can be combined and enriched by additional lexical information:

(35b)

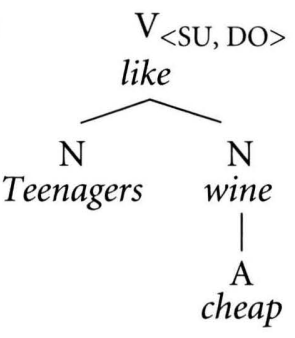

Finally, the dependency relations that exist between the word forms can be further specified on dependency lines (cf. Mel'čuk 1988: 16ff.; 2003: 189ff.):

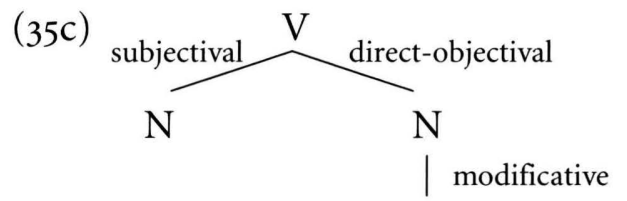

A

It is not part of the system of basic dependency principles that the highest element is a verb, but in many classical dependency representations this is the case. As a result, the whole tree is a verbal node (a VP) identical with the sentence, which is thus an endocentric construction. Even if the subject (as a macro-complement) is seen as dependent on verb inflection (= the subject as a micro-complement) rather than the lexical verb (Eroms 2000), endocentricity is preserved.

The diagrams (35) to (35c) do not show the word order in the sentence. This was seen as an advantage by followers of Tesnière, since dependency representations do not aim at the linear but at the structural order of the sentence. However, dependency diagrams can be arranged in a projective manner (cf. Heringer 1996; Eroms 2000):

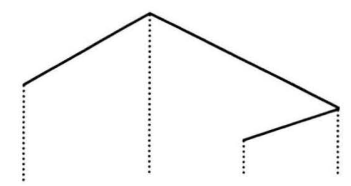

Teenagers like cheap wine.

\subsubsection{Foundation of dependency}

There are two aspects to the foundation or justification of dependency. On the one hand, there is the fundamental question of the empirical foundation and 
psychological reality of dependency relations. In this respect, DG has received impressive external justification (Leiss 2003). On the other hand, there is the theory internal justification of "above" and "below" in a dependency structure: how can it be determined for (structurally, i.e., non-trivially) co-occurring elements? Which of them is the regent (head) and which one the dependent? We will now introduce relation types, which were used in attempts to operationalize dependency (=D) (cf. Welke 1995):

D1 Endocentricity: the node building potential of a word category (= regent). In most cases the regent is thus a precondition for the occurrence of the dependent.

D2 Sub-categorization: the regent implies a set of dependents, determining their syntactic and semantic functions.

D3 Government: the regent determines certain formal features (case, status, canonical position, prosody).

D1 applies to optional elements in particular:

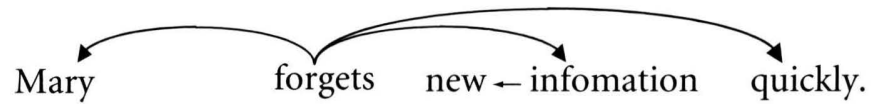

The occurrence of the noun information is a precondition for the occurrence of the adjective new. The occurrence of the verb forget is a precondition for the occurrence of the adverb quickly. In both cases the dependent can be omitted, but not the regent. D1 has problems, if both elements are obligatory and thus presume each other, e.g., the car ${ }^{11}$ or with her. The same applies to the occurrence of forget: although the verb is a precondition for the occurrence of the nouns Mary and information, the opposite also applies. As English structurally requires macro-realization of subject and objects, the occurrence of the two nouns is a precondition for the occurrence of the verb forget. According to D1, one would have to assume bidirectional dependency (interdependency) between the verb and its complementation frame. But this is not permitted by the basic principles that we have introduced.

The problem is solved by D2: the verb implies the occurrence of the two nouns since it belongs to the sub-category of divalent verbs. This means that valency relations are used to justify the direction of dependencies: valency carriers are also governing elements in a dependency tree. But not all dependencies that occur in a sentence are implied (adjuncts, particles). Thus a dependency tree based solely on D2 would remain incomplete. $\mathrm{D}_{3}$ supports some of the dependencies established 
by $\mathrm{D} 1$ and $\mathrm{D} 2$, but can also contradict them $\left(\leftarrow_{\text {form }}=\right.$ form government; cf. 10.2.3.2 a) ii-iv): ${ }^{12}$

(37) French belles $\leftarrow$ form filles

(38) Latin Vicino $_{\text {Dat }} \leftarrow$ form favet. to-neighbor favor-(s)he.

'(S)he favors the neighbor.'

(39) Persian kûhe $\leftarrow$ form boländ (Nichols 1986: 58 )

mountain high

'high mountain'

(40) English The car $\rightarrow_{\text {form }}$ drives well.

Belles in (37) is according to D1 dependent on filles 'girls', but also according to $\mathrm{D}_{3}$, as the gender of filles (feminine) determines the feminine form of the adjective (belles, not beaux). Vicino in (38) is according to D2 dependent on favet, but also according to $\mathrm{D}_{3}$, as favere 'to favor' determines the case of vicino (dative). In (39) the adjective boländ is according to D1 dependent on kûhe, but the existence of an attribute has the effect that the noun $k \hat{u} h$ receives the suffix $-e$ : thus boländ form-governs kûhe. According to D2, the noun car in (40) is dependent on drives, but the singular car causes drives to be marked as singular, thus car form-governs drives. Generally, regent or head marking as in (39) and (40) runs contrary to the dependency relations that D1 and D2 establish. If dependency relations are solely based on morphological government, the direction of the relation changes according to the morphological linguistic type (regent vs. dependent marking). Also, a purely morphological dependency notion does not establish a complete tree, since not all elements would be connected to each other (Melč'uk 1988: 109):

(41) French $\mathrm{Il} \rightarrow_{\text {form }} \mathrm{a} \rightarrow$ form dormi dans sa $\leftarrow_{\text {form }}$ chambre.

'He has slept in his/her room'

Finally, form-government relations can be complex and running in opposite directions, depending on the participating grammatical categories: in a German noun node such as den $_{\text {Acc }}$ billigen $_{\text {Acc }}$ Wein ('the cheap wine'), for which it is usually assumed that the determiner (den) is the structural regent (head) and the noun (Wein) is the lexical regent (core), three different form-government relations $(=\mathrm{R})$ can be identified:

R1 (type: case):

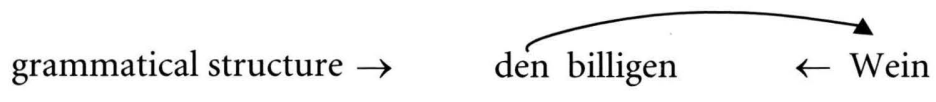

12 We restrict discussion to (a) the (more common) notion of form government and (b) to the discussion of government that is morphologically marked. In principle, form government includes the determination of topology and prosody. 
The top regent inside the phrase or node (= the internal governor) is the structural regent (head). It directly governs the lexical regent (core), indirectly the adjective. The case is externally determined: the structural regent itself is governed, its regent can be found in the respective grammatical structure. It is primarily the structural regent that is morphologically marked (den vs. die, das).

R2 (type: grammatical gender):

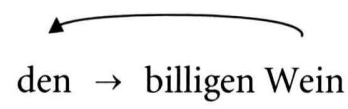

The internal governor is the lexical regent (core). It directly governs the structural regent (head), indirectly the adjective. The grammatical gender is internally determined as it is an inherent grammatical feature of the noun (here: masculine). It is primarily the structural regent that is morphologically marked (den vs. die, das).

$\mathrm{R}_{3}$ (type: number):

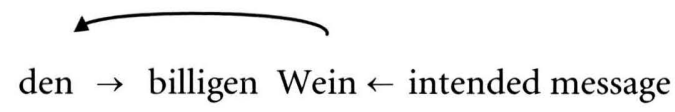

The internal governor is the lexical regent (core). It directly governs the structural regent (head), indirectly the adjective. Number is externally determined, but not through government: speakers freely choose number according to the message they intend to convey. It is primarily the lexical regent that is morphologically marked (Wein vs. Weine).

According to which of the three Rs is considered, different structural descriptions result, all of which can be defended. Thus we can see that the choice of the internal governor depends on the perspective that is chosen for the description:

- If one focuses on the abstract case relation, the structural regent (head) is the internal governor.

- If, on the other hand, the abstract gender or number relation was focused on, it is the lexical regent (the noun) that is the internal governor.

- If the concrete morphological marking of the syntagmatic relations is focused on then in relation to case and gender, it is the determiner that is the internal governor, but in relation to number, it is the noun.

- Finally, if the way the respective category is determined is focused on, phrase (node)-internal determination, i.e., the gender relation, would rank highest in selecting the internal governor. Among the external factors, determination through government (case relation) would rank higher than the free choice according to the communicative intention (number relation). Accordingly, there would not just be a dichotomy (+/- internal governor (head)), but a tripartite 
division from maximum via medium to minimum support for being the internal governor (headedness). ${ }^{13}$

We have discussed three possible ways of founding or justifying dependency. None of the three defines a complete dependency tree in the sense of a pure DG: D1 leads to interdependencies, $\mathrm{D}_{2}$ and $\mathrm{D}_{3}$ leave elements isolated, $\mathrm{D}_{3}$ divides into the independent relations $\mathrm{R}_{1}-\mathrm{R}_{3}$. In practice, dependency grammarians tend to work with multirelational dependency concepts.

\subsubsection{Dependency und constituency}

Dependency und constituency were first seen as competing means of representation. Gaifman (1965) showed that both were "weakly equivalent": chains generated by a projective dependency grammar can also be generated by a phrase structure grammar. Apart from the rather restrictive basis for the comparison, the result does not mean that dependency and constituency make identical structural claims. We will show that the opposite is the case. Our discussion draws on Engel (1994: 23ff.) and Matthews (1981: 71ff., 2007: 112ff.) (see also Fischer 1997: 13ff.; Uzonyi 2003).

Dependency and constituency are based on connexions (rule-governed co-occurrences). Constituency shows that elements that are connected by connexions form a bigger whole.

(42)

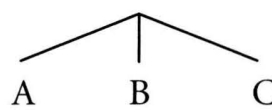

(constituency)

Dependency shows connexions directly:

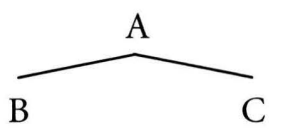

(dependency)

The first fundamental criticism of constituency by DG is that connexions are not shown directly but only indirectly via the constituent in which elements linked by connexions are a part. Although it is the relation between the elements that is primary and constituents are thus only derived from this relation, constituency representations turn the derived datum into the primary datum.

The constituency representation (42) is more complex than the dependency representation (43). (42) claims connexions between all three elements; a dependency representation of three elements can only show two connexions. The greater complexity of (42) is only an advantage if all three connexions in fact exist. Generally it

\footnotetext{
13 For additional information on the structure of the German noun node, see Ágel (2006).
} 
is a problem for constituency representations that their assumptions are too strong. Constituency representations permit further differentiation:

(44)

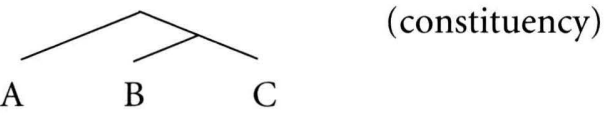

In (44), it is claimed that A enters a connexion with the BC-grouping. In a pure DG, there is only limited scope for representing connexions between elements and groupings of elements:

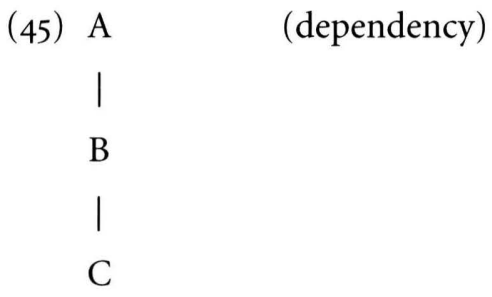

Like (43), (45) only shows two connexions, but $\mathrm{C}$ is also indirectly dependent on A (principle of transitivity). Thus BC forms a node with the nucleus A. Working from the bottom of a branch upward, groupings can be read off a dependency representation. However, this is not possible if $\mathrm{AB}$ forms a grouping. Such a structure could be assumed for German adjective declension (cf. den billigen Wein), since it is both determined by the structural (head) and the lexical regent (core) (cf. R1$\mathrm{R}_{3}$ in 10.3.3). The problem can be solved by making the additional assumption that a dependency structure can act as the nucleus of a node (cf. Lobin 1993): $(\mathrm{A} \rightarrow \mathrm{B}) \rightarrow \mathrm{C}$. We can summarize our discussion up to now by stipulating that in a comparative assessment of dependency and constituency representations the achievements should be weighed against the representational cost.

The second fundamental criticism of constituency by DG is that constituency does not allow a natural representation of a regent (head), while DG is all about a direct representation of the regent. Compare two common constituency analyses with two common dependency representations:

(46) $[[$ very old $]$ books $] \quad$ vs. $\quad$ very $\leftarrow$ old $\leftarrow$ books

(47) $[$ no $[$ fresh bread $]] \quad$ vs. $\quad$ no fresh $\leftarrow$ bread

It is not possible to formulate a procedure that derives the respective regents (heads) from the two constituency representations. Regents have to be identified using additional descriptive means, for instance by introducing the X-bar scheme and the dependency relations c-command and m-command and thus a convention that only allows regents (heads) to be non-phrases, while c- or m-commanded dependent elements must be phrases. 
But constituency representations, too, contain information that cannot be shown in a dependency representation: one cannot derive from the dependency representation in (47) that the dependency structure of fresh bread itself has nuclear qualities.

We note that dependency and constituency do not merely represent connexions differently but also make different structural statements: the concept of a grouping is as alien to dependency as is the concept of a regent (head) to constituency. Therefore the two means of representation should be seen as complementary. Dependency representations might include constituency relations at certain points, while generative grammar has, with the introduction of the X-bar scheme and the relations of c-command and $\mathrm{m}$-command, in effect included dependency relations in its model. These developments have somewhat defused the controversy about the better basic means of representation and point to a converging development (cf. already Schmidt 1991).

As we mentioned in 10.1.4, a different convergent development, the integration of projectionist and constructionist model components, is to be expected (see also 10.2.5 and 10.2.7). It is the syntactic theory that can solve both convergence tasks most convincingly that, in the more distant rather than the immediate future, will be able to lay claim to being the best theory. 